\title{
IMAGE COMPRESSION TECHNIQUES USING ARTIFICIAL NEURAL NETWORK
}

\author{
YOGESH CHANDRA ${ }^{1 *}$, SHIKHA MISHRA ${ }^{2}$ \\ ${ }^{* I}$ M.TECH SCHOLAR,${ }^{2}$ FACULTY OF ELECTRONICS \& COMMUNICATION , \\ *1,2Dr. C.V.RAMAN INSTITUTE OF SCIENCE \& TECHNOLOGY \\ *19406209025, *1 chandray187@gmail.com, ${ }^{2} 9827470038,{ }^{2}$ shikha.mishra687@gmail.com
}

Corresponding Author:-

EmailID - chandray187@gmail.com,9406209025,

\begin{abstract}
: -
This paper contain neural network bases on image compression techniques.The image compression is the application of the data compression on digital images. The main role of the image compression technique which is used to reduce the number of pixels and reduce the broadcast and transmission cost in efficient form and an artificial neural network is a computational structure which is stimulated by observed process in neural network of biological neuron in the brain. Image compression based on back propagation neural network with Liebenberg - marquardt algorithm and it is achieved by image is divided into number of blocks and select the one neural network for each block according to its complexity value. The Training algorithm and back propagation neural network is used to increase the performance and to decrease the convergence time and provide high compression ratio and low distortion. The neural network training process best for image compression and applications.
\end{abstract}

Keywords : - Artificial neural network, Back propagation neural network, image Compression, L-M algorithms;

\section{(c) $(\$)$}




\section{INTRODUCTION}

Image Processing is a very popular for image compression and day to day improvement of the image compression. Image processing is the display of graphical image, manipulation, analysis and storage of the images. An artificial neural networks are increasingly being examined and [1] considered as possible solutions to problems and for application in many fields where high computation rates are required [2]. Using Artificial neural network (ANN) technique with various image compression methods [3] [4] [5] [6]. A detail survey of about how ANN can be applied for compression purpose is report in [7] [8] [9] [10].Broadly, two different categories for improving the compression methods and performance have been suggested. The existence method of compression by using ANN techniques so that improvement in the design of existing method can be achieved and apply neural network to develop the compression scheme itself, so that new methods can be developed and further research and possibilities. The classical image compression methods are based on the BPNN techniques. The Back propagation Neural Network (BPNN) is the most commonly used in multi-layer feed forward ANN. The BPNN consists of three or more fully interconnected layers of neurons. The BP training can be applied to any multilayer NN that uses differentiable activation function and supervised training [11]. The BPNN has the simplest architecture of ANN that has been developed for image compression but its drawback is very slow convergence. In [12] suggested mapping the gray levels of the image pixels and their neighbors in such a way that the difference in gray levels of the neighbors with the pixel is minimized and then the CR and network convergence can be improved. In [13] used BPNN for image compression and developed algorithm based on improved BP. The blocks of original image is number of blocks and consider the features of intensity change. In [14] image compression adaptive method based on BPNN for image compression is divided into number of blocks based on complexity level, computing the complexity of each block and then selecting one network for each block according to its complexity value. The rest of this paper is organized as follows: Section II describes the image compression technique. Section III presents the Artificial Neural Network using back propagation neural network. Section IV covers the proposed methodology. In Section V mat lab result and graphs are discussed. Finally, Section IV concludes the paper.

\section{IMAGE COMPRESSION}

Image compression is the application of data compression on digital images. The main objective of image compression is to reduce redundancy of the image pixels in the suitable form of storage media or transmit data (e-mail) in an competent form. The basic two types of image compression:

Lossy: Lossy compression is a data compression method which loses some of the data from original data which is result being a smaller file size. Most common files type like JPG and BMP.

Lossless: Lossless compression is a data compression method there are no loss of compressed data that is it allows for a smaller file size, but also decompressed back to the original size and qualities. Most common file types like TIFF, PSD,

PNG, GIF, and RAW. Image compression focuses on the problem of reducing the amount of data required to represent a digital image. The Process of reducing the image for image storage requirements of representation of an image. Compression is obtained by the removal of one or more of the three basic data redundancies: When less than Coding redundancy is optimal code words are used. Inter pixel redundancy results from correlations between the pixels of an image. Psycho visual redundancy is due to data that is ignored by the human visual system. Image compression techniques is used to reduce the number of bits required by representing an image and taking advantage of these redundancies. An inverse process called decompression (decoding) is applied to the compressed data to get reconstructed image [15].The objective of compression is to reduce the number of bits as possible, when keeping the resolution and the visual quality of the reconstructed image. Image compression process is composed of two distinct blocks: an encoder and a decoder. Image $\mathrm{F}(\mathrm{x}, \mathrm{y})$ is fed into the encoder, which is created a set of symbols form the input data and uses them to represent the image

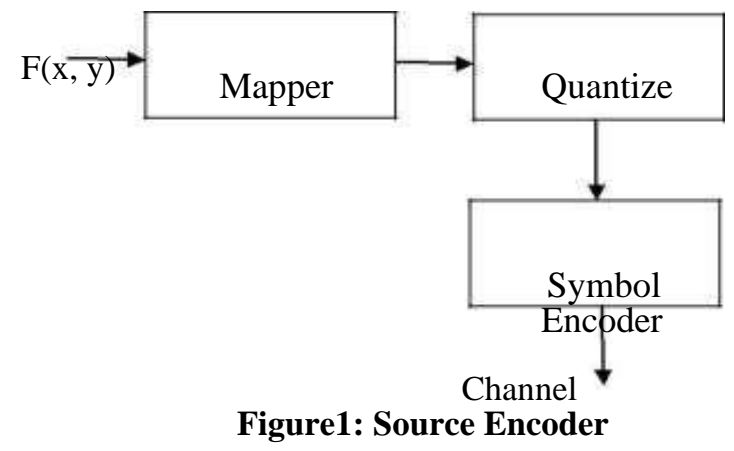

Let us $\mathrm{n} 1$ and $\mathrm{n} 2$ denote the number of information carrying units in the original and encoded images respectively, the compression that is achieved can be quantified numerically via the compression ratio[16].

The encoder is responsible for reducing the coding; inter pixel and psycho visual redundancies of input image. In first stage, the mapper which is work on the transforms the input image into a format calculated to reduce inters pixel redundancies. 


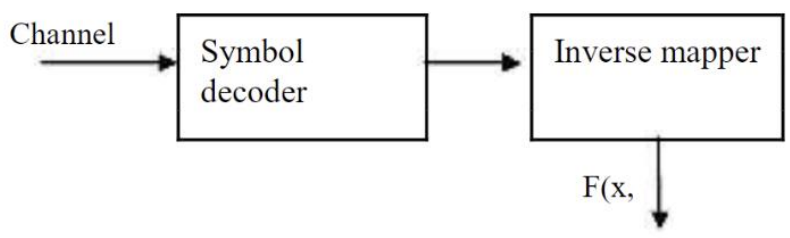

Figure2: Source Decoder

The second stage, quantized block is to reduce the accuracy of mapper's output in according to defined criteria. In third and last stage, a symbol decoder is used to create a code for quantize output and maps the output in according to the code. These blocks perform in reverse process, the reverse operation of the encoder's symbol coder and mapped block. In quantization process is irreversible; an inverse process is not included.

\section{III.ARTIFICIAL NEURAL NETWORKS}

An Artificial Neural Network (ANN) is an information-processing theory that is inspired which is the way of biological nervous systems, just like as the brain process information. The key element of this concept is the fresh structure of the information processing system.

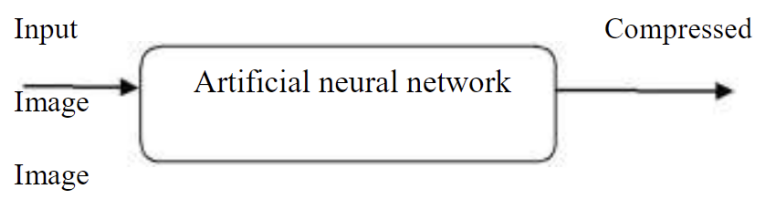

Figure3: Basic image compression

The artificial neural network is composed of a large number of greatly interconnected of processing elements (are called neurons) working in union to solve particular problems [17]. An ANN is configured for a particular application, such as through a learning process, pattern recognition or data classification, Learning in biological systems including the adjustments of the synaptic connections that exist among the neurons. The multiple layers of simple processing elements are known as neurons. Each neuron is linked to sure of its neighbors with changing coefficients of connectivity that represent the strengths of these connections.

\section{A. BACK PROPEGATION NEURAL NETWORK}

Three layers, one input layer, one output layer and one hidden layer, are assigned. Both of input layer and output layer are fully connected to hidden layer. Compression is obtained by designing the value of the number of neurons in the both input layer and output layers neuron less than the hidden layer neuron. The neural network can be either linear or nonlinear network according to the transfer function working in the layers. The Log-sigmoid function is one of the most common functions given in equation(1) working in different neural networks Problems.

$$
\mathrm{f}(\mathrm{x})=\frac{1}{1+\exp (-\mathrm{x})}
$$

Some neural networks employs log-sigmoid in the hidden layer and linear in the output layer. It is nonlinear functions have a more capability of learning both linear and nonlinear problems than linear ones. [18] The output of the jth neuron in the hidden layer is given by

$$
\mathrm{Zj}=f 1\left(\sum_{i=1}^{n} w i j x i+b j\right)
$$

The kth neuron of output Yk in the output layer is

$$
\mathrm{yk}=f 2\left(\sum_{l=1}^{m} w k l z l+b k\right)
$$

Where $\mathrm{f} 1$, $\mathrm{f} 2$ is the hidden layer activation functions and the output layer respectively, wji in eqn(2) is the synaptic weight connecting the ith input node to the jth neuron of the hidden layer, bj is the bias in the hidden layer of the jth neuron and $\mathrm{n}$ is the number of hidden layer neurons, $\mathrm{fl}$ is the activation function, and $\mathrm{Zj}$ is the output in the hidden layer.

\section{IV.PROPOSED METHODOLOGY}

\subsection{L-M ALGORITHM}

The Levenberg- marquadt algorithm and two layer feed forward neural network was considered. A feed forward neural network is used to image coding consists of the following steps are: An image, I, is divided into blocks of pixels then scan each block in the form a input vector $\mathrm{x}(\mathrm{n})$ of size

$$
\mathrm{p}=\text { row }^{*} \text { column }
$$


It is supposed that the hidden layer of the layer network consists of Q neurons each with W synapses, and it is considered by selected weight matrix Wh. All number of blocks of the original image is passed through the hidden layers to obtain the hidden signals, $h(n)$, which represent encoded input image blocks, $x(n)$. If $Q<W$ such coding delivers image compression [19]. It is assumed that the output layer consists of the $\mathrm{m}=\mathrm{p}=\mathrm{row}^{*}$ column; neuron with synapses.

Let Wy be an appropriately particular output weight matrix. All $\mathrm{N}$ hidden vector $\mathrm{h}(\mathrm{n})$ and expressive an encoded image Ie, are passes through the output layer to get the output signal $y(n)$. The output signals are reassemble into $p=$ row*column; image blocks to get a reconstructed image, Ir. There are two error matrices is used to measure the different image compression. Firstly the Mean Square Error (MSE) and the second Peak Signal-to- Noise Ratio (PSNR). The MSE is the cumulative squared error between the original image and the compressed image. The peak error measured by the PSNR .

$$
\mathrm{mse}=\mathrm{i} / \mathrm{mn} \sum_{\mathrm{y}=1}^{\mathrm{m}} \sum_{\mathrm{x}=1}^{\mathrm{n}}\left[\mathrm{I}(\mathrm{x}, \mathrm{y})-\mathrm{I}^{\prime}(\mathrm{x}, \mathrm{y})\right]_{2}
$$

The Peak signal-to-noise ratio (PSNR) defined as

$$
\text { PSNR }=10 \log 10[255 / \text { sqrt }(\mathrm{MSE})]
$$

The training is conduct for using the Levenberg -Marquardt algorithm is a representative class of images. The weight matrices have select, by using the Wh matrix of any image can be quickly encoded, and then decoded using the Wy matrix.

\subsection{ALGORITHM STEPS}

Step1: Read the input image.

Step2: Image is divided into number of blocks. Step3: Each blocks scanning for complexity level. Step4: Initialization of neurons.

Step5: Apply each neuron on the input layer from scan vectors.

Step6: Execute the operation depending on the weights and the logic involve.

Step7: Passes them to the hidden layer. Step8: Repeat the same as in step6. Step9: Reassemble the outputs.

Step10: Neural network training and wait the weights.

\subsection{TRAINING PROCEDURE}

The data from an image is encoded into a construction of the hidden and output weight matrices by the training procedure It is assumed that an image, I, used in training of size Rx C and consists of blocks row*column.

The first step is to convert a block of matrix I into a matrix X of size P x N contain training vectors, $\mathrm{x}$ (n), from image blocks. $\mathrm{P}=$ row $^{*}$ column and p.N = R.C

The second step is the target data is equal to the made data=X and the last step is the network is trained until the mean squared error(MSE) is small. The matrices Wh and Wy will be used in the image encoding and decoding steps.

Image Encoding: The hidden-half of the two-layer network is used to encode images. This method can be defined as follows: I->X, H= (Wh. X),

Where $\mathrm{X}$ is an encoded image of I. Image Decoding: Using the output-half the two-layer network is the decoded image and it is defined as follows: $\mathrm{Y}=(\mathrm{Wy} . \mathrm{H}), \mathrm{Y}-\mathrm{I}$.

\section{MATLAB RESULTS AND GRAPHS}

The simulation results for images $(64 \times 64)$ are shown in below and performance measure graphs are also included. Considered the image, the better compression performance with highest $\mathrm{CR}$, the least back propagation rate, and highest PSNR [20].

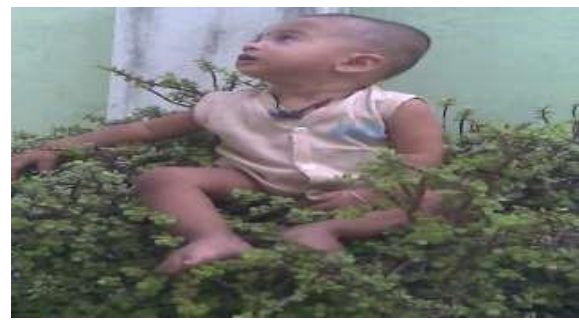

Figure4: Original image

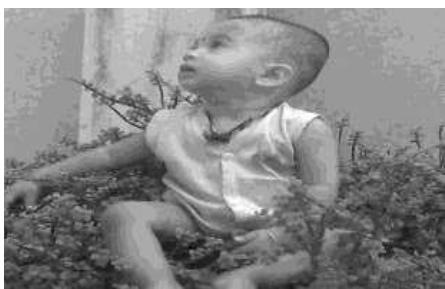

Figure5: Compressed image 
The performance measure graph and experimental results are shown in below fig6.

\section{VI.CONCLUSION}

In image compression technique convergence time also play main role for quality of image and the back propagation is very popular for image compression technique. The back propagation neural network and levenberg-marquardt algorithms estimating a connection counted that image compression and convergence time can be improved. This paper contains image compression techniques using back propagation neural network with levenberg-marquardt algorithm and calculate the peak signal to noise ratio and compression ratio for image compression

\section{REFERENCES}

[1]. R. P. Lippmann, “An introduction to computing with neural network”, IEEE ASSP mag., pp. 36-54, 1987.

[2]. M.M. Polycarpou, P. A. Ioannou, "Learning and Convergence Analysis of Neural Type Structured Networks", IEEE Transactions on Neural Network, Vol 2, Jan 1992, pp.39-50.

[3]. Rao, P.V. Madhusudana, S.Nachiketh,S.S.Keerthi, K. "image compression using artificial neural network".EEE, ICMLC 2010, PP: 121-124.

[4]. Dutta, D.P.; Choudhury, S.D.; Hussain, M.A.; Majumder, S.; "Digital image compression using neural network" .IEEE, international Conference on Advances in Computing, Control, Telecommunication Technologies, 2009. ACT '09.

[5]. N.M.Rahim, T.Yahagi, "Image Compression by new sub-image bloc Classification techniques using Neural. Networks", IEICE Trans. On Fundamentals, Vol. E83-A, No.10, pp 2040-2043, 2000.

[6]. M. S. Rahim, "Image compression by new sub- image block Classification techniques using eural network". IEICE ,E83-A (10), (2000), pp. 2040- 2043.

[7]. D. Anthony,et.al. "A study of data compression using neural networks and principal component analysis, "in Colloquium on Biomedical Applications of Digital Signal Processing,1989, pp. 1-5.

[8]. G. L. Sicuranzi, G. Ramponi, and S. Marsi, “Artificial neural network for image compression,”Electronics Letters, vol. 26, no. 7, pp. 477-479, March 291990.

[9]. M.Egmont-Petersen, D.de.Ridder, Handels, “Image Processing with Neural Networks - a review”,Pattern Recognition 35(2002) 2279-2301 [11] M. H. Hassoun, Fundamentals of Artificial NeuralNetworks, MIT Press, Cambridge, MA, 1995.

[10]. Wasserman, P.D., 1989. Neural Computing: Theory and Practice. Coriolis Group, New York, USA,ISBN: 10: 0442207433, pp: 230.

[11]. Durai S.A. And E.A. Saro, 2006. Image compression with back-propagation neural network usingcumulative distribution function. World Acad. Sci. Eng. Technol., 17: 60-64.

[12]. Xianghong, T. And L. Yang, 2008. An image compressing algorithm based on classified blockswith BPNN. Proceeding of ICCSSE, Dec. 12-14, IEEE Computer Society, and Wuhan, Hubei pp: 819-822.

[13]. Veisi, H. And M. Jamzad, 2009. A complexity-based approach in image compression using neuralnetworks. Int. J. Sign. Process. 5: 82-92.

[14]. B. M. Wilamowski, Y. Chen, A. Malinowski, "Efficient algorithm for training neural networks with one hidden layer," In Proc. IJCNN, vol.3, pp.1725-728, 1999.

[15]. SubramanyaA,'Image compression Technique" s IEEE, Vol. 20, Issue 1, pp 19-23, Feb-March2001.

[16]. E. Watanabe and K. Mori, "Lossy Image Compression Using a Modular Structured Neural Network," Proceed- ings of IEEE Signal Processing Society Workshop, Wash- ington DC, 2001, pp. 403-412.

[17]. S .S. Panda1, M.S.R.S Prasad2, MNM Prasad3, Ch. SKVR Naidu4” Image Compression Using Back Propagation Neural Network" IJESAT Vol-2,Issue - 1, 74 -78. Jan-feb-2012.

[18]. Pranob KCharles1et.al."Artificial Neural Network based Image Compression using LevenbergMarquardt Algorithm"IJMERVol.1, Issue.2, pp-482-489 ISSN:2249-6645.2010.

[19]. ying chulet.at.'Image Compression Using Multilayer Neural Networks based on Fast Bacterial Swarming Algorithms"Proceedings of the 7th International Conference on Machine Learning and Cybernetics, Kunming, 1215July2008.

[20]. Charif, H. Nait, and Salam, Fathi M.,"Neural networks-based image compression system," Proc. 43rd IEEE Midwest symp. On circuits and systems, Lansing MI, August 2000.

[21]. Luo Lincong, Feng Hao, Ding Lijun”Color Image Compression Based on Quaternion Neural Network Principal ComponentAnalysis"978-1-4244-7874-3/10 2010 IEEE.

[22]. Vilas Gaidhane1 , Vijander Singh2, Mahendra Kumar3" Image Compression using PCA and Improved Technique with MLP Neural Network" 2010 ICARTCC 978-0-7695-4201-0/10 \$26.00 @ 2010 IEEE.

[23]. Sonal, Dinesh Kumar" a study of various image compression techniques"

[24]. Licheng Liu, "The progress and analysis of image compression based on BPANN Microcomputer Information, Vol 23, No. 6, pp. 312-314, Feb. 2007.

[25]. G. W. Cottrell and ottrell and p. munro, "Principal Component Analysis of images via back propagation," IN SPIE VOL. 1001 Visual communication and image processing '88, 1988, PP. 1070-1077.

[26]. N. Sonehars, M. Kawato, S. Miyake, and K. Nakane, "Image data compression using a neural network model”, Proc. of IJCNN, Washington DC, pp. 35-41, Oct.1989.

[27]. O. AbdeI-Wahhab, and M. M. Fahmy, "Image compression using multilayer neural networks", IEE Proc.-Visual Image Signal Processing, Vol. 144, No. 5, pp. 307-312, Oct. 1997 
[28]. Xiangyang Liu, Ruyun Wang, and Wei Li, "Image compression based upon new back propagation", Computer Applications and Software, Vol. 12, No. 5, pp. 14-15,71, May. 2004.

[29]. R. C. Gonzales, R. E. Woods, "Digital Image Processing", Second Edition, Prentice-Hall, 2002.uter Science \& Engineering Survey (IJCSES) Vol.1, No.2, November 2010.

[30]. G.L. Sicuranza, G. Ramponi, S. Marsi, "Artificial neural network for image compression", Electronic letters 26, 477$479,1990$. 